\title{
Study on the Key Technology of Micro-arc Oxidation of TA2 Titanium for Medical Use
}

$$
\begin{gathered}
\text { Yu-lei Ll }{ }^{a, *} \text {, Yue-lai DAl }{ }^{b} \text {, Jin-jun TANG }{ }^{c}, \text { Xiao-yu } \text { JIN }^{d} \text { and Qun } \\
\text { WANG }^{e}
\end{gathered}
$$

Ningbo Sub-academy of the National Weapons Science Research Academy, Ningbo 315103, China

aliyulei7395@163.com, 'daiyuelai_1988@126.com, 'jinjun.tang@163.com, dinxiaoyu_nb@163.com, ewangqun_yz@163.com

${ }^{*}$ Corresponding author

Keywords: TA2, micro-arc oxidation, Film thickness, Surface morphology.

\begin{abstract}
By micro-arc oxidation technology, the TA2 titanium metal surface modification, by scanning electron microscopy (SEM) experiments, the effects of different voltage parameters on film thickness and surface microstructure were studied. The results show that as the voltage increases and the oxidation time increases, the thickness of the film increases and the surface roughness increases.
\end{abstract}

\section{Introduction}

Medical TA2 pure titanium materials, with its good biocompatibility, and excellent corrosion resistance, comprehensive mechanical and process properties in a physiological environment, has been more and more widely used in clinic, gradually become artificial knee joint, dental implants, root and denture metal stents, etc. ${ }^{[1]}$ human hard tissue repairable and substitute materials. However, pure titanium TA2 in organisms, usually in the form of biological inert, does not have biological activity, with the combination of bone is a kind of mechanical lock. After implant implantation in the human body, the material direct contact with tissue, cell or blood for a long time, the surface characteristics have an impact to the tissue around the implant, which will be likely to cause organism, toxicity and inflammatory reactions ${ }^{[2]}$ and thrombosis, affect the patients health and life safety. These are seriously restricted its further development ${ }^{[3-4]}$.Therefore, the titanium surface modification ${ }^{[5]}$ to improve its biological activity is necessary. Micro-arc oxidation calcium phosphate coating is the most promising application value. In this paper, the ceramic coatings of TA2 were prepared by micro-arc oxidation in the electrolyte containing calcium and phosphorus, and the effects of different voltage and oxidation time on the surface morphology were studied.

\section{Experiment}

Experimental materials using pure titanium TA2 materials, its size is $15 \mathrm{~mm} * 15 \mathrm{~mm} *$ $3 \mathrm{~mm}$, in turn, using 400 \#, 600 \#, 800 \#, 1000 \#, 1200 \# metallographic sandpaper to no obvious scratches. With anhydrous ethanol, acetone and deionized water ultrasonic cleaning 10 to 30 minutes, putting the sample into the oven in $40{ }^{\circ} \mathrm{C}$ drying. Sample as the anode and stainless steel as cathode, alkaline calcium phosphate salt solution as the micro-arc oxidation electrolyte, use different voltages and oxidation time to experiment. In this experiment, the film thickness and the morphology of the surface film were observed by scanning electron microscopy. 
Put sample in with a good solution, gradually increase the voltage between the poles, with the increase of voltage, metallic titanium surface gradually disappear. After the experiment, the sample surface is pale, with coating effect, as shown in Fig.1.

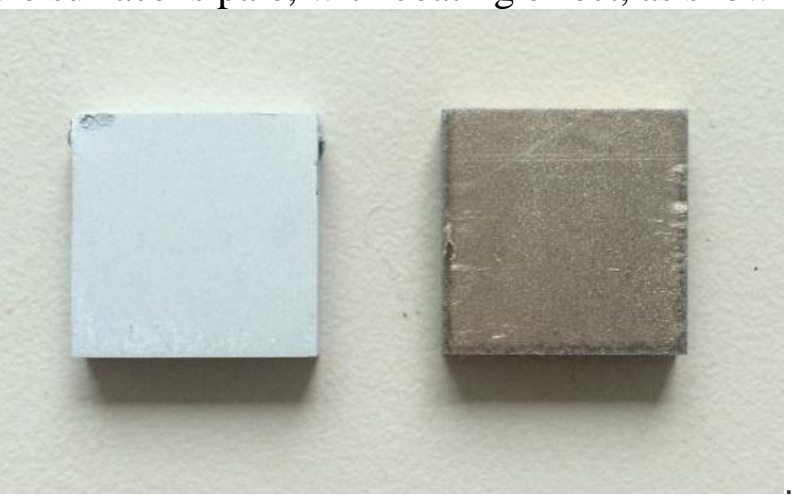

Fig.1.Before and after the experiment sample contrast

\section{Results and Discussion}

Table 1: experiment results of medical TA2 titanium micro-arc oxidation process membrane layer thickness

\begin{tabular}{|c|c|c|c|}
\hline serial number & Voltage (V) & Oxidation time (min) & $\begin{array}{c}\text { Membrane layer } \\
\text { thickness(mm) }\end{array}$ \\
\hline 1 & 320 & 5 & 43 \\
\hline 2 & 360 & 5 & 51 \\
\hline 3 & 400 & 5 & 55 \\
\hline 4 & 360 & 10 & 56 \\
\hline 5 & 400 & 10 & 59 \\
\hline
\end{tabular}
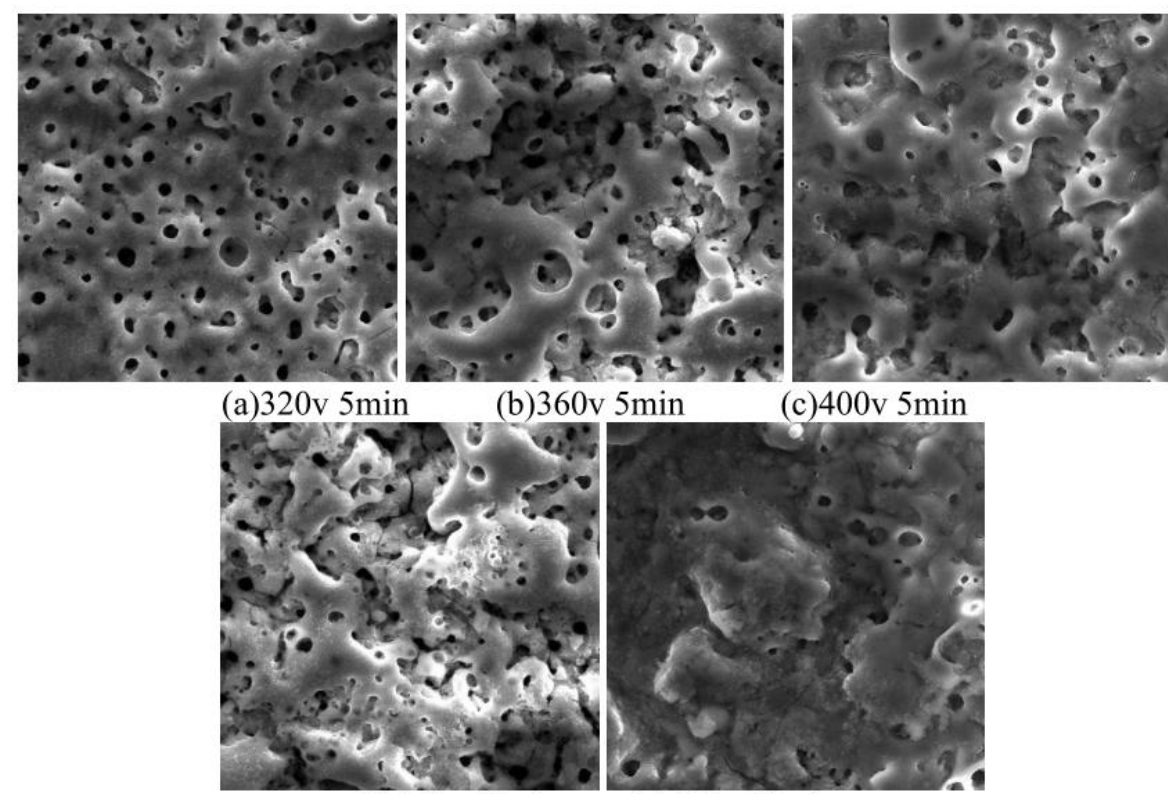

(d) $360 \mathrm{v} 10 \mathrm{~min}$

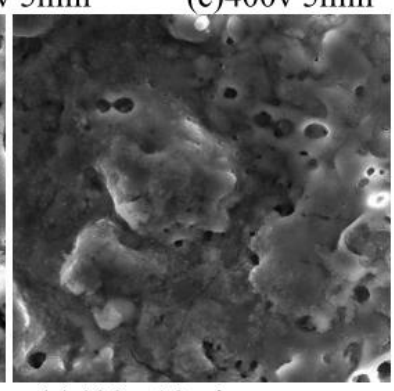

(e) $400 \mathrm{v} 10 \mathrm{~min}$

Fig.2.different technological parameters of micro-arc oxidation ceramic layer surface morphology $(1000 x)$ 


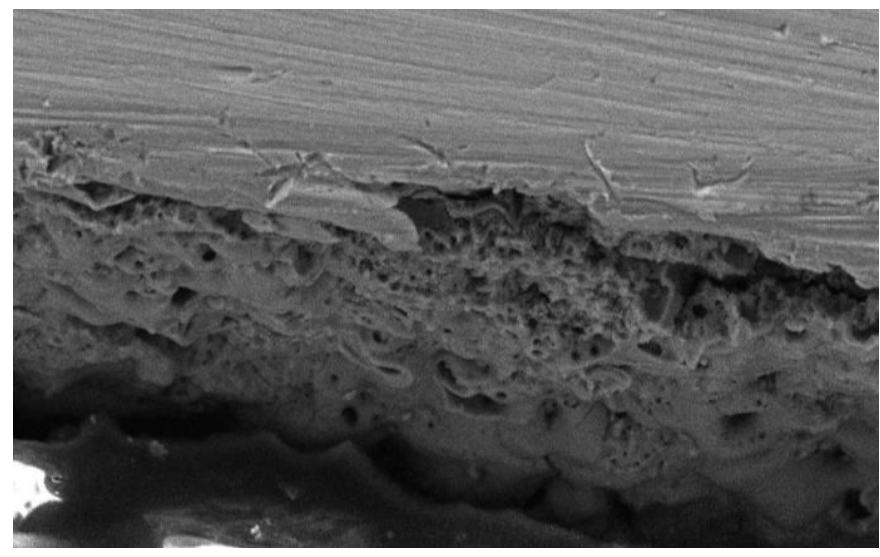

Fig.3.Micro-arc oxidation ceramic layer cross-sectional morphology (600x)

As shown in Fig.2, the surface of the film after micro-arc oxidation was covered with micropores. Uneven surface pore distribution, pore size varies, about 0.5-5um in diameter, stacked staggered, like crater. As shown in Fig.3, the film has a certain thickness, and the thickness direction of the pores.

In the chemical process, electrochemical process and the plasma chemical processes, the surface of the specimen formatted discharge. Ion collision will happen in the discharge area, the process is quick and with the exothermic effect and volume expansion. Due to the high temperature and high pressure in the discharge channel, the molten reaction product is ejected from the channel. After they encounter the solution, they are cooled at an extremely fast rate and are solidified around the discharge channel holes. While the product in the channel is also cooled and contracted, so that a laminated porous topography can be formed on the surface. It can be seen from Fig.2 that there are small cracks and non-smooth phenomena on the hole wall. This is caused by the concentration of the electrolyte. With the increase of the concentration, the discharge increases, and the surface heat release of the titanium metal increases. At this point the internal and external temperature of the solidification increased, prone to internal stress, resulting in cracks around the hole wall.

First of all, under the condition of the same electrical parameters, the sample do voltage of $320 \mathrm{v}, 360 \mathrm{v}, 400 \mathrm{v}$ micro-arc oxidation treatment. As shown in Table 1, it can be seen that the thickness of the oxide film with the increase of voltage is constantly thickening, and the thickness of the growth rate shows the tendency of decrease. Initial stage of micro-arc oxidation, oxidation film forming quickly, outward growth is mainly composed of membrane layer, micro-arc discharge began, oxide film layer is dissolved, breakdown channels form accumulation position from the melt, the higher the voltage, the more energy of breakdown, the greater the accumulation of the melt, the faster of thick film growth rate, but as the membrane layer thickening, oxide film breakdown difficultly. Seen from Fig.2, the film's surface gradually become coarse with the increase of the voltage, because the surface quality of the oxide is determined by the energy of the single pulse discharge. As the voltage increases, the unit energy increases and the discharge intensity increases. So the exothermic effect of the oxide also increased. At this time it has more energy by the thermal effect and volume expansion, resulting in larger pores and more accumulation around micropores. Therefore, the bigger the voltage, the rougher the surface.

Then, under the condition of the same electrical parameters: the sample time about 5 min, 10 min, respectively do the micro-arc oxidation treatment. As shown in Table 1, the thickness of the oxide film is thickening over time, constantly in the process of 
gradually thickening, porosity is declining, and the film-forming rate of $5 \mathrm{~min}$ before is significantly greater than 5 min after. In the process of micro-arc oxidation, the growth of the oxide film is a dynamic equilibrium process. The film thickness mainly depends on the oxygen in the discharge channel. With the increase of the thickness of the oxide film, the conductivity decreases. The film is more and more difficult to break, the breakdown voltage is also increasing, resulting in slower growth rate. It speculates that after the film thickness reaches a certain degree, the film growth rate is close to zero, the thickness of the film will reach a maximum. On the other hand, as the processing time is prolonged, the amount of outward growth of the film becomes less, mainly inward oxidation, resulting in increased film density and reduced porosity. This process exacerbates the unevenness of the film and the surface becomes more rough.

\section{Conclusion}

Within the scope of the test, as the voltage increases, TA2 titanium material calcium phosphate membrane layer thickness increases, the surface roughness increases.

Within the scope of the test, as the extension of oxidation time, TA2 titanium material calcium phosphate film thickness increases and the surface roughness increases.

\section{References}

[1] Geetha M,Singh AK,Asokamani R et al. Ti based biomaterials, the ultimate choice for orthopaedic implants-A review[J]. Progress in Materials Science,2009,54(3): 397-425.

[2] Hallab NJ,Anderson S,Stafford T,et al. Lymphocyte responses in patients with total hip arthroplasty[J]. Journal of Orthopaedic Research,2005,23(2): 384-391.

[3] Tian Y S, Chen C Z, Li S T et al. Applied Surface Science[J],2005, 242: 177.

[4] Du Nan,Wang Shuaixing,Zhao Qing,et al. Microstructure and tribological properties of microarc oxidation composite coating containing Cr2O3 particles on TC4 titanium alloy[J]. Rare Metal Materials and Engineering,2013,42(3): 621-624.

[5] LIU X J,LI G,XIA Y. Investigation of the discharge mechanism of plasma electrolytic oxidaon using Ti tracer[J]. Surface and Coatings Technology,2012,206(21): 4462-4465. 